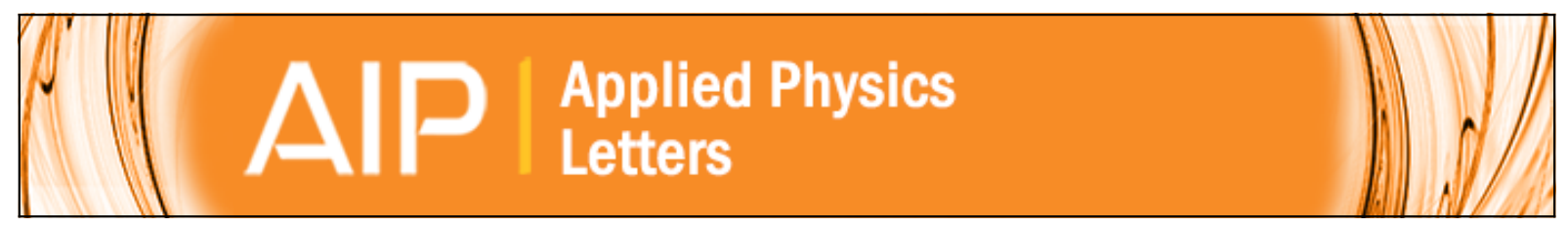

\title{
Magnetic force microscopy of the submicron magnetic assembly in a magnetotactic bacterium
}

\author{
R. B. Proksch, T. E. Schäffer, B. M. Moskowitz, E. D. Dahlberg, D. A. Bazylinski, and R. B. Frankel
}

Citation: Applied Physics Letters 66, 2582 (1995); doi: 10.1063/1.113508

View online: http://dx.doi.org/10.1063/1.113508

View Table of Contents: http://scitation.aip.org/content/aip/journal/apl/66/19?ver=pdfcov

Published by the AIP Publishing

\section{Articles you may be interested in}

Nanomechanical properties of the sea-water bacterium Paracoccus seriniphilus-A scanning force microscopy approach

Biointerphases 10, 019004 (2015); 10.1116/1.4906862

Micrometric periodic assembly of magnetotactic bacteria and magnetic nanoparticles using audio tapes J. Appl. Phys. 111, 044905 (2012); 10.1063/1.3681380

Quantitative analysis of magnetization reversal in submicron S -patterned structures with narrow constrictions by magnetic force microscopy

Appl. Phys. Lett. 86, 053111 (2005); 10.1063/1.1853491

Instrument for the measurement of hysteresis loops of magnetotactic bacteria and other systems containing submicron magnetic particles

Rev. Sci. Instrum. 72, 2724 (2001); 10.1063/1.1361079

Submicron characterization of recording media using magnetic force microscopy (invited) (abstract)

J. Appl. Phys. 79, 6440 (1996); 10.1063/1.361965

\section{AP $\mid$ APL Photonics \\ APL Photonics is pleased to announce Benjamin Eggleton as its Editor-in-Chief}

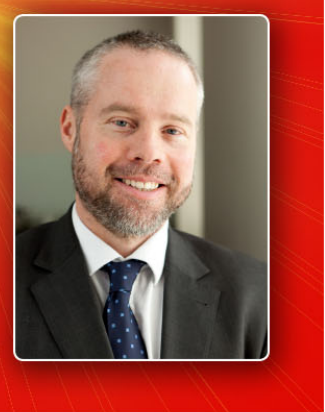




\title{
Magnetic force microscopy of the submicron magnetic assembly in a magnetotactic bacterium
}

\author{
R. B. Proksch ${ }^{\mathrm{a})}$ and T. E. Schäffer \\ Department of Physics, University of California at Santa Barbara, Santa Barbara, California 93106 \\ B. M. Moskowitz \\ Institute for Rock Magnetism, University of Minnesota, Minneapolis, Minnesota 55455 \\ E. D. Dahlberg \\ Magnetic Microscopy Center, University of Minnesota, Minneapolis, Minnesota 55455 \\ D. A. Bazylinski \\ Marine Science Center, Northeastern University, East Point, Nahant, Massachusetts 01908 \\ R. B. Frankel \\ Department of Physics, California Polytechnic State University, San Luis Obispo, California 93407
}

(Received 20 September 1994; accepted for publication 21 February 1995)

\begin{abstract}
A magnetic force microscope (MFM) was used to image topography and magnetic forces from a chain of submicron single magnetic domain particles produced by and contained in isolated magnetotactic bacteria. The noncontact magnetic force microscope data were used to determine a value for the magnetic moment of an individual bacterial cell, of order $10^{-13} \mathrm{emu}$, consistent with the average magnetic moment of bacteria from the same sample, obtained by superconducting quantum interference device magnetometry. The results represent the most sensitive quantification of a magnetic force microscope image to date. (c) 1995 American Institute of Physics.
\end{abstract}

Magnetic force microscopes have been used for high resolution imaging of a variety of samples of interest in micromagnetism. However, the potential of MFMs has yet to be realized because of the difficulty of quantifying the magnetic field the MFM measures. Some progress towards quantification has occurred, ${ }^{1}$ but has been limited primarily by the uncertainties in the micromagnetics of the specimens. As a step towards overcoming this limitation, we have quantified the response of a MFM to a simple micromagnetic system consisting of a linear chain of single magnetic domain particles within a magnetotactic bacterium. The geometrical simplicity of the particle chain facilitates the quantification process because it was possible to estimate the total magnetic dipole moment of the chain assembly by simply measuring the chain length. This estimate provided the starting point for a nonlinear model of the MFM image. The fitted moment resulting from the nonlinear model agreed well with the average moment estimated from magnetic measurements on a bulk sample of the bacteria.

Magnetotactic bacteria mineralize intracellular magnetosomes, which are membrane-enclosed, single-magneticdomain particles of magnetite, $\mathrm{Fe}_{3} \mathrm{O}_{4}$, or greigite, $\mathrm{Fe}_{3} \mathrm{~S}_{4}{ }^{2}$ The particles are characterized by a narrow size distribution and species-specific crystalline habit. Magnetosomes are arranged in one or more linear chains along the symmetry axis of the cell, which constitutes a permanent magnetic dipole in the cell. The torque exerted by the ambient magnetic field on the permanent cellular dipole causes the bacterium to be oriented and to migrate along the magnetic field lines, a phenomenon referred to as magnetotaxis. For this study, the

\footnotetext{
${ }^{a)}$ Permanent address: Department of Physics, St. Olaf College, Northfield, MN 55057. Electronic mail: proksch@ @hysics.ucsb.edu
}

magnetotactic bacterial strain MV-1 were grown and harvested as previously described. ${ }^{3}$ Cells were fixed with $1 \%$ gluteraldehyde and freeze dried. The freeze drying process insured the magnetosomes were close to the surface of the cell, simplifying the magnetic imaging. The freeze-dried cells had a coercivity of 385 Oe at room temperature. Individual magnetite particles in strain MV1 are truncated hexahedral prisms with average dimensions of $53 \times 35 \times 35 \mathrm{~nm}$ and organized in a single linear chain of 10-25 particles. ${ }^{4}$ Using a SQUID magnetometer, ${ }^{5}$ the average moment per bacterium was determined to be $1.6 \times 10^{-13}$ emu at $300 \mathrm{~K}$ agreeing with previous measurements of the moments of magnetotactic bacteria. ${ }^{1,6}$

To correlate the magnetic field measurement with an individual cell, it was necessary to obtain the topographic and associated magnetic images of the magnetotactic bacterium with the same cantilever. A Nanoscope III from Digital Instruments was used in the "tapping mode" graphic image of the cell. The cantilever was then retracted from the surface and a noncontact magnetic force mode image was taken over the same area of the sample. This process of alternating short-range topographical and long-range magnetic images was repeated several times. A lateral drift of only a few nanometers between successive topographic images was observed. Figure 1(a) is a tapping mode image of the cell topography; the rough surface features are evidently effects of the freeze drying procedure. Figure 1(b) is the MFM image over the same area as Fig. 1(a).

The long-range magnetic interactions between the tip and the sample affect the mechanical behavior of the cantilever. When the magnetic perturbation is small and constant over the range of the cantilever motion, it can be shown that 


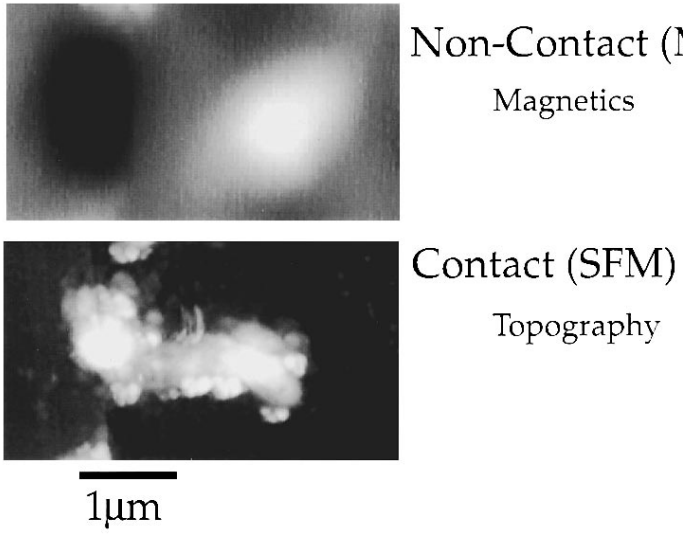

FIG. 1. (a) A contact mode image of a freeze-dried MV-1 cell is shown on the bottom. The cell has experienced significant lysing from the freezedrying process. (b) On the top is a MFM image taken immediately following the contact mode image. The MFM image was taken at a height of $50 \mathrm{~nm}$ above the surface of the bacteria. Dipolar fields originating from the freezedried cell are clearly visible in this image.

the magnetic forces cause changes in the amplitude of the cantilever $\Delta A$, given by ${ }^{1,8}$

$$
\Delta A=\left.\frac{\partial A}{\partial \omega_{D}}\right|_{\omega_{D}^{\max }} \frac{1}{2 k} \int_{\text {Tip Volume }} \mathbf{m}(\mathbf{r}) \cdot \frac{\partial^{2} \mathbf{B}}{\partial z^{2}}\left(\mathbf{r}-\mathbf{r}^{\prime}\right) d^{3} r^{\prime} .
$$

Here, $\partial A /\left.\partial \omega_{D}\right|_{\omega_{D}^{\max }}$ is the experimentally determined slope of the amplitude versus drive frequency curve and the drive frequency $\omega_{D}$, which is usually chosen to maximize the MFM response, $k$ is the measured spring constant of the cantilever, $\mathbf{r}$ and $\mathbf{r}^{\prime}$ are vectors defined in Figs. 2(b), $\mathbf{m}\left(\mathbf{r}^{\prime}\right)$ is the tip magnetization and the integral is taken over the volume of the magnetic material coating the cantilever tip. ${ }^{1}$

As seen in Eq. (1) the response of a MFM depends on a number of experimental parameters, including the magnetic state of the tip and the sample. To determine the magnetic state of the tip we used a field emission electron microscope to determine the shape, measured the bulk properties of the $\mathrm{CoCr}$ magnetic film used to coat the cantilevers, and fit MFM traces to the average fields expected from bit transitions in a thin-film magnetic hard disk. From this process, it was estimated that the magnetic film coating on the tip had a magnetization of $M_{s}^{\text {tip }}=720 \mathrm{emu} / \mathrm{cm}^{3}$, was magnetized primarily perpendicular to the tip surface [see Fig. 2(a)], and had a coercivity of approximately 300 Oe, large enough so that field from a cell was not sufficient to reverse the tip

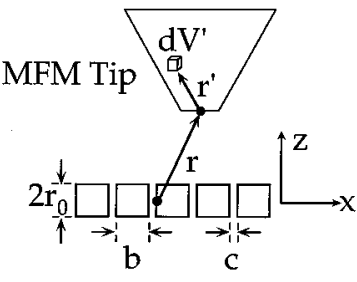

a)

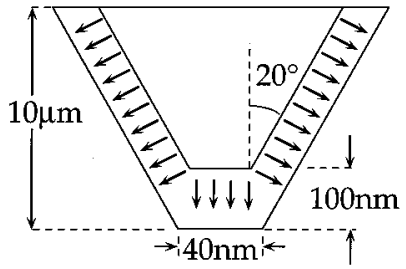

b)
FIG. 2. (a) The model of the MFM tip and chain of magnetosomes used in this work. (b) Details of the tip model including an illustration of the tip magnetization.

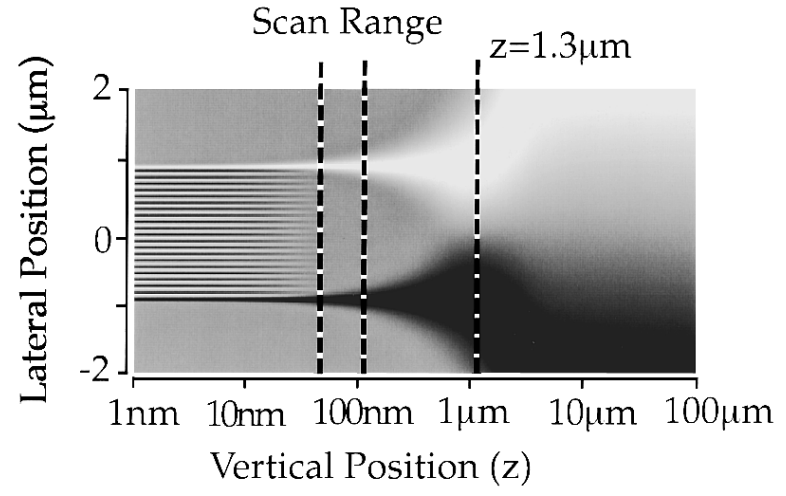

FIG. 3. This is a gray scale image of $\partial^{2} B_{z} / \partial z^{2}$ above a chain of 21 magnetosomes. The magnitude of the field is denoted by color, white being positive and dark being negative. The values of the field have been scaled by the maximum magnitude of the field at a particular height to maximize the contrast at that height. The range of heights possible for our MFM tip is indicated in the figure as "Scan Range". $z=1.3 \mu \mathrm{m}$ is also labeled. Below this value, the lateral distance between the minimum and maximum of the field is equivalent to the length of the chain of magnetosomes.

magnetization. The shape of the tip was determined by electron microscopy to be a truncated pyramid with an angle of $20^{\circ}$ and a height of $10 \mu \mathrm{m}$. The radius of the tip was $20 \mathrm{~nm}$. Similar tip dimensions have been reported by other investigators. ${ }^{9}$ The spring constant of the cantilever was measured to be $k=0.8 \mathrm{~N} / \mathrm{m} .{ }^{10}$

The separation between the tip and the surface of the freeze dried cell was determined by the $z$-piezo calibration to be $50 \mathrm{~nm}$. Because of the chain of magnetosomes resided at some unknown distance below the cell surface, this number was considered a minimum value for the tip-magnetosome separation. The tip-sample separation was therefore included in the analysis as a nonlinear fitting parameter constrained to be between 50 and $120 \mathrm{~nm}$.

For modeling the MFM image of the magnetosomes, it was necessary to calculate the spatial derivatives of the magnetic field produced by the magnetosome chain. The magnetosomes were modeled as a chain of uniformly magnetized cylinders [see Fig. 2(b)] because the shape closely matched the actual magnetosomes and was easy to calculate. We used a cylinder length of $b=50 \mathrm{~nm}$, a radius of $r_{0}=17.5 \mathrm{~nm}$, and a magnetosome separation of $c=10 \mathrm{~nm} .^{5}$ Assume the magnetosomes were magnetized along the axis of cylindrical symmetry which allow the field to be calculated by modeling an individual magnetosome as two oppositely magnetostatically charged disks. Furthermore, because the MFM tip was always several disk radii away from the magnetosome chain, we found it convenient to expand the magnetostatic potential of a charged disk in terms of Legendre polynomials and calculate the field from $\mathbf{B}=\nabla \Phi_{M} \cdot{ }^{11}$

A grayscale image of $\partial^{2} B_{z} / \partial z^{2}$ from a chain containing 21 magnetosomes using our expansion is shown in Fig. 3. The magnitude of the field for a given $z$ has been normalized to the maximum field value at that height. This allows the effective contrast to be observed as a function of $z$ even though the magnitude changes by roughly ten orders of magnitude from a distance of $1 \mathrm{~nm}$ to $1 \mathrm{~mm}$.

A first estimate of the magnetic moment of the chain of 


\section{Fitting for Cell \#1}

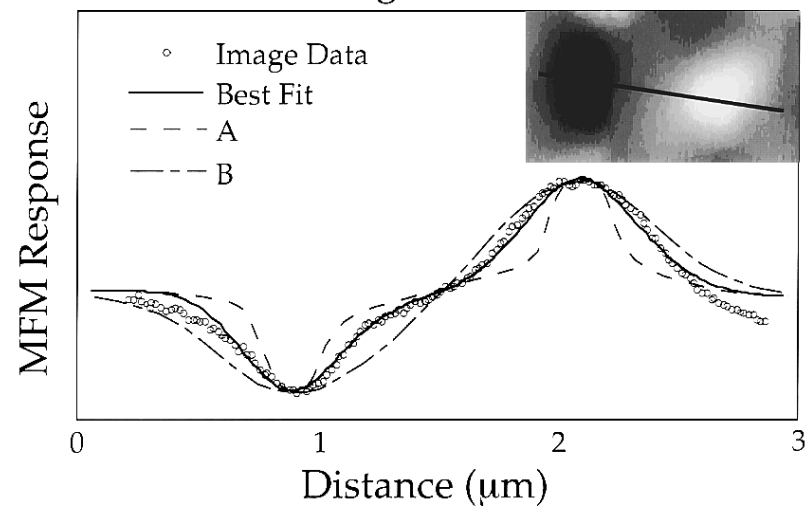

FIG. 4. Results of fitting the MFM tip model to the dipolar signal from the magnetotactic bacteria cell in Fig. 1. An inset from Fig. 1 shows the portion of the image the data for fitting was extracted from. The fitted moment was $4 \times 10^{-13}$ emu for this cell. The tip-sample separation used in the calculation of the best fit was $65 \mathrm{~nm}$. The effects of varying this parameter are shown in curves A and B where a tip-sample separation of 50 and $100 \mathrm{~nm}$, respectively, were used.

magnetosomes comes from a simple geometric observation. From Fig. 1(a), the distance between the minimum and the maximum in the MFM image is $1.25 \mu \mathrm{m}$. A careful inspection of Fig. 3 reveals that the distance between the minima and the maxima of the $z$ component of the field derivative corresponds to the total length of the magnetosome chain if $z<1.3 \mu \mathrm{m}$. In this limit the max-min value can be used to determine the actual length of the chain of magnetosomes. This and TEM measurements of the dimensions of the magnetosomes $(b=50 \mathrm{~nm}$ and $c=10 \mathrm{~nm})$ constrains the number of magnetosomes to be 21 , justifying our original choice. Assuming the magnetosomes are uniformly magnetized, single domain magnetite particles $\left(M_{S}=480 \mathrm{emu} / \mathrm{cm}^{3}\right)$ the total moment of the chain is calculated to be $1.2 \times 10^{-12}$ emu. This is a value 7.5 times larger than the bulk average.

Using the above estimate of the cellular moment as a staring point, a nonlinear Levenberg-Marquardt ${ }^{12}$ fitting routine was employed to further refine the measurement of the magnetic moment from the nanoscale magnetic assembly. The fitting procedure yielded a moment for the bacterial cell of $4 \times 10^{-13}$ emu with a tip-sample separation of $65 \mathrm{~nm}$. This value for the moment is more than a factor of three smaller than the geometrical estimate and closer to the value obtained from the bulk magnetization measurements. The results of this fitting are shown in Fig. 4. The effects of varying the tip-sample separations between 50 and $100 \mathrm{~nm}$ are shown in curves A and B of Fig. 4. We repeated this procedure for two cells with smaller signals and found the fitted moments to be $1 \times 10^{-13}$ and $2 \times 10^{-13}$ emu, respectively.

We have presented a procedure for the quantification of the MFM image of a magnetotactic bacterium. The procedure was iterative, starting with a model of the system based on TEM measurements and then refined with a nonlinear fitting routine. For systems where there is no a priori magnetic information, quantification of MFM images will require an accurate determination of the tip to specimen distance, and of the magnetic state of the sensing tip. Although use of bits in a hard disk are convenient for the latter, it may be necessary to calibrate the system using a better defined micromagnetic system such as these magnetotactic bacteria.

Other magnetic microscopies may also provide useful information, especially scanning SQUIDs and Hall probes which are capable of very sensitive field measurements and have the advantage of giving direct field values rather than gradients. While the lateral resolution of a SQUID ${ }^{13}$ is insufficient to resolve the field from a magnetotactic bacterium, it is possible that a scanning Hall probe might have both the sensitivity and lateral resolution. ${ }^{14}$

This work also represents the first direct magnetic measurement of the magnetic dipole moment of a magnetotactic bacterium. The fact that the final fitted moment was smaller than that estimated from the length of the chain, could be due to gaps in the chain, or to micromagnetic effects such as curling of the moments in the particles to reduce the magnetostatic energy. Further study will be required to clarify this point. In any case, it is clear that MFM can be a useful tool for the study of magnetotactic bacteria and other nanoscale magnetic structures.

We thank K. Babcock, J. P. Cleveland, M. Dugas, and P. K. Hansma for helpful suggestions. R. P. and E. D. D. were supported by the Office of Naval Research Grant N0001491-J-1290, B. M. M. was supported by the National Science Foundation. This is Contribution 9403 of the Institute of Rock Magnetism. D. A. B. and R. B. F. were supported by the Office of Naval Research Grant No. N00014-91-J-1290.

${ }^{1}$ P. Grütter, H. J. Mamin, and D. Rugar, in Scanning Tunneling Microscopy II, edited by R. Weisendanger and H.-J. Güntherodt (Springer-Verlag, Berlin, 1992), pp. 151-207, and references therein.

${ }^{2}$ D. A. Bazylinski, A. J. Garrett-Reed, and R. B. Frankel, Microsc. Res. Technol. 27, 389 (1994).

${ }^{3}$ D. A. Bazylinski, R. B. Frankel, and H. J. Jannasch, Nature (London) 334, 518 (1988).

${ }^{4}$ N. H. C. Sparks, S. Mann, D. A. Bazylkinski, D. R. Lovely, H. W. Jannasch, and R. B. Frankel, Earth. Planet Sci. Lett. 98, 14 (1990).

${ }^{5}$ MPMS-5 from Quantum Design Inc., San Diego, CA.

${ }^{6}$ C. Rosenblatt, F. Flavio Torres de Araujo, and R. B. Frankel, Biophys. J. 40, 83 (1982)

${ }^{7}$ Digital Instruments, Santa Barbara, CA.

${ }^{8}$ R. Proksch, M. Radmacher, T. Schaeffer, and P. K. Hansma (unpublished).

${ }^{9}$ K. Babcock, V. Elings, M. Dugas, and S. Loper, IEEE Trans. Magn. 30, 4503 (1994).

${ }^{10}$ J. P. Cleveland, S. Manne, D. Bocek, and P. K. Hansma, Rev. Sci. Instrum. 64, 403 (1993).

${ }^{11}$ J. D. Jackson, Classical Electrodynamics, 2nd ed. (Wiley, New York, 1975).

${ }^{12}$ W. H. Press, B. P. Flannery, S. A. Teukolsky, and W. T. Vetterling, $\mathrm{Nu}-$ merical Recipes (Cambridge University Press, Cambridge, 1986).

${ }^{13}$ J. R. Kirtley, M. B. Ketchen, K. G. Stawiasz, J. Z. Sun, W. J. Gallagher, S. H. Blanton, and S. J. Wind, Appl. Phys. Lett. 66, 1138 (1995).

${ }^{14}$ H. Hallen, R. Seshadri, A. M. Chang, R. E. Miller, L. N. Pfeiffer, K. W. West, C. A. Murray, and H. F. Hess, Phys. Rev. Lett. 71, 3007 (1993). 